Acta Crystallographica Section D

Biological

Crystallography

ISSN 0907-4449

Renée Ménez, ${ }^{a}$ Nicholas G. Housden, ${ }^{\text {b }}$ Stephen Harrison, ${ }^{b}$ Colette Jolivet-Reynaud, ${ }^{c}$ Michael G. Gore ${ }^{\mathrm{b}}$ and Enrico Adriano Stura ${ }^{d}$ *

a Département R\&D Immuno-essais et Protéomique, BioMerieux, Marcy l'Etoile, France, 'b Institute of Biomolecular Sciences, University of Southampton, Bassett Crescent East, Southampton SO16 7PX, England, ' Unité Mixte de Recherche, CNRS/BioMérieux, Centre d'Etudes et de Recherche en Virologie et Immunologie, Lyon, France, and ${ }^{\mathbf{d}}$ Département d'Ingénierie et d'Etudes des Protéines (DIEP), C.E. Saclay, 91191 Gif-sur-Yvette, France

Correspondence e-mail: estura@cea.fr

Received 18 September 2004 Accepted 3 March 2005

\section{Different crystal packing in Fab-protein L semi-disordered peptide complex}

Proteins and peptides with variable degrees of disorder are a challenge for protein crystallization. These may be completely disordered or just contain regions with a high degree of mobility that may be represented by a multitude of discretely defined conformations. These difficulties are not insurmountable, but it may be unreasonable to expect a clean result from a structural point of view. The complex between a murine monoclonal antibody (19D9D6) and a synthetic peptide that encompasses the first 45 residues of the core protein of Hepatitis $\mathrm{C}$ virus that is poorly structured in solution has been crystallized. In order to make the crystallization possible, use was made of a single immunoglobulin-binding domain of protein L from Peptostreptococcus magnus ( $\mathrm{PpL}$ ), a bacterial protein that can bind the variable region $(\mathrm{Fv})$ of a large population of antibodies through its light chain with no interference with antibody-antigen recognition. Crystals were obtained in different space groups where the size of the cavity that accommodates the peptide is different, although many of the crystal contacts and the overall lattice are preserved. The peptide can be considered to be semidisordered and the larger cavity accommodates a better ordered peptide than the smaller one. The lattice is of interest for the design of a scaffold system for the crystallization of peptide-tagged proteins since a cavity that accommodates a disordered entity might be able to host ordered proteins of the same size and shape as the cavity. Here, the differences between the lattices formed by this trimolecular complex are described and it is discussed how such a system may be adapted to the crystallization of peptide-tagged proteins.

\section{Introduction}

Hepatitis $\mathrm{C}$ virus ( $\mathrm{HCV}$ ) is an enveloped single-stranded RNA virus and the major causative agent of transfusion-associated hepatitis. It encodes a polyprotein which is post-translationally cleaved into structural and non-structural proteins among which is the core protein (amino acids 1-191) that forms the nucleocapsid of the virion. The rapid antigen response elicited by the highly conserved core antigen at the onset of the disease is widely used to screen for HCV infection (Park et al., 1995). Monoclonal antibody 19D9D6 was generated by immunizing mice with the truncated recombinant $1-120$ region (Jolivet-Reynaud et al., 1998). Recently, we have reported the X-ray crystallographic analysis of the complex between Fab 19D9D6 and a 28 -amino-acid peptide (residues 13-40 of core) at $2.34 \AA$ resolution (Ménez et al., 2003). The minimal recognized epitope is QIVGGVYLL (residues 29-37 of core) and only this part of the peptide is well ordered in electron density. Most studies have been focused on the longer peptide 2-45. This peptide has been studied by nuclear magnetic resonance (NMR) and molecular modelling and shown to have a propensity for a small three-dimensional motif composed of two $\alpha$-helixes separated by a loop, the rest of the peptide being disordered (PDB code 1cwx; Ladavière et al., 1997). This motif was not found in the crystal structure. In order to ensure that the different conformation was not an artefact of the shorter peptide we have crystallized the complex between Fab' 19D9D6 and the peptide 2-45. This longer peptide has the inconvenience that at high concentrations it has a strong tendency to aggregate as shown by competition ELISA experiments (Ménez et al., 2003). Although aggregation per se does not prevent crystallization (Stura, Martin et al., 2002), it remains problematic and certain attempts at obtaining 
crystals of the complexes resulted in crystals of the free Fab' 19D9D6. However, by screening against an array of $\mathrm{PpL}$ mutants, trying various stoichiometric ratios of $\mathrm{Fab}^{\prime}$ and antigen and allowing the crystallization to proceed slowly, we found two lattices that contained the peptide. Following the same procedure, we have also obtained crystals of the 120-residue recombinant immunization antigen which has a much stronger propensity for aggregation. Here we report the details of the crystallization, the crystal structure of the Fab' 19D9D6 complex with peptide 2-45 and PpL and discuss the conformation adopted by the peptide and how it depends on the crystal packing.
Table 1

Data collection and structure determination.

\begin{tabular}{|c|c|c|c|c|}
\hline Data set & 2-45 D55A/L57H/Y64W & 2-45 D55A/L57H/Y64W & 2-45 H74C/Y64W & Core 120 H74C/Y64W \\
\hline Crystallization & $\begin{array}{l}\text { 9\% MPEG 5K, } \\
\text { NaOAc pH } 4.5\end{array}$ & $\begin{array}{l}\text { 8\% MPEG 5K, } \\
\text { NaOAc pH } 4.5\end{array}$ & $\begin{array}{l}10.3 \% \text { MPEG } 5 \mathrm{~K}, \\
0.5 \mathrm{M} \mathrm{NaCl}, \\
0.2 \mathrm{M} \text { Tris- } \mathrm{HCl} \mathrm{pH} 9.0\end{array}$ & $\begin{array}{l}\text { 10.3\% MPEG } 5 \mathrm{~K}, \\
0.5 \mathrm{M} \mathrm{NaCl}, \\
0.2 \mathrm{M} \text { Tris- } \mathrm{HCl} \mathrm{pH} 9.0\end{array}$ \\
\hline Cross-seeding & Yes (L55/Y64W) & No & Yes $(\mathrm{L} 57 \mathrm{H})$ & No \\
\hline Data collection & ESRF ID14EH1 & ESRF ID14EH2 & ESRF ID29 & ESRF BM30A \\
\hline Space group & $P 2_{1}$ & $P 2_{1} 2_{1} 2$ & $P 2_{1} 2_{1} 2$ & $P 2$ \\
\hline \multicolumn{5}{|l|}{ Unit-cell parameters } \\
\hline$a, b, c(\AA)$ & $43.6,230.5,123.6$ & $129.5,222.47,43.8$ & $129.3,222.9,43.6$ & $94.7,43.2,104.6$ \\
\hline$\alpha, \beta, \gamma\left({ }^{\circ}\right)$ & $90.0,91.7,90.0$ & $90.0,90.0,90.0$ & $90.0,90.0,90.0$ & $90.0,117.9,90.0$ \\
\hline Resolution range $(\AA)$ & $20.0-3.5$ & $20.0-3.05$ & $20.0-2.59$ & $20.0-3.2$ \\
\hline Completeness (\%) & 99.4 & 89.5 & 99.5 & 97.8 \\
\hline Multiplicity & 3.5 & 3.15 & 3 & 3.5 \\
\hline$\langle I / \sigma I\rangle$ & 2.0 & 5.4 & 5.2 & 10. \\
\hline$R_{\mathrm{sym}}(\%)$ & 17.3 & 9.8 & 10.5 & 9.7 \\
\hline Asymmetric unit & $4 \mathrm{Fab}^{\prime}+$ & $2 \mathrm{Fab}^{\prime}+$ & $2 \mathrm{Fab}^{\prime}+$ & $1 \mathrm{Fab}^{\prime}$ \\
\hline Content & $\begin{array}{l}2 \text { peptides }+ \\
1 \text { shared } 4+\mathrm{PpL}\end{array}$ & $\begin{array}{l}2 \text { peptides }+ \\
2 \mathrm{PpL}\end{array}$ & $\begin{array}{l}2 \text { peptides }+ \\
2 \mathrm{PpL}\end{array}$ & $\begin{array}{l}1 \text { shared } \mathrm{C} 120+ \\
1 \mathrm{PpL}\end{array}$ \\
\hline Refinement & СCP4 & $\mathrm{CCP} 4$ & $\mathrm{CCP} 4$ & $\mathrm{CCP} 4$ \\
\hline$R_{\text {free }}(\%)$ & 28.5 & 30.1 & 24.0 & 29.1 \\
\hline$R_{\text {work }}(\%)$ & 16.9 & 19.1 & 19.9 & 20.0 \\
\hline PDB code & $1 \mathrm{xcq}$ & $1 \mathrm{xct}$ & $1 \times f 5$ & \\
\hline
\end{tabular}

\section{Materials and methods}

\subsection{Preparation of Fab' 19D9D6, peptide synthesis and expression} of core 120

Murine monoclonal antibody 19D9D6 was raised against the first 120 residues of $\mathrm{HCV}$ core antigen, it is an $\mathrm{IgG} 1, \mathrm{~V}_{\mathrm{L}} \kappa_{9}$ (JolivetReynaud et al., 1998). This light chain binds well to protein L. It was cultured, sequenced, expressed, cleaved and purified as described in detail elsewhere (Ménez et al., 2003). Briefly, the IgG was purified on a protein A column and then cleaved with pepsin to yield $\mathrm{F}\left(\mathrm{ab}^{\prime}\right)_{2}$. The $\mathrm{F}\left(\mathrm{ab}^{\prime}\right)_{2}$ were purified by size-exclusion and reduced to $\mathrm{Fab}^{\prime}$ fragments with mercaptoethanol followed by the blocking of the free cysteines with iodoacetamide. The $\mathrm{Fab}^{\prime}$ was then purified by size-exclusion chromatography. Peptide $2-45$ was synthesized on an automatic synthesiser, purified and confirmed by amino-acid analysis and mass spectroscopy as described in Ménez et al. (2003). Region 1-120 of HCV-1a core was expressed as a fusion protein with a hexahistine peptide in Escherichia coli and purified by metal-chelation chromatography on an Ni-NTA column (Jolivet-Reynaud et al., 1998).

\subsection{Crystallization}

The complex with peptide 2-45 was prepared in solution in advance of the crystallization trials, by mixing $40 \mu \mathrm{l}$ of Fab' 19D9D6 $\left(6 \mathrm{mg} \mathrm{ml}^{-1}\right)$ with $3 \mu \mathrm{l}$ of peptide $\left(20 \mathrm{mg} \mathrm{ml}^{-1}\right)$. Screening for crystallization was carried out by sitting-drop vapour diffusion using Q-plates and using multiple drops per reservoir so that many different PpL mutants could be tested simultaneously over the same reservoir (Stura, 1999a, 2001). The screening and the optimization experiments were carried out at $290 \mathrm{~K}$ in an air-conditioned room. Only two precipitant solutions used in the crystallization of other Fab' 19D9D6 complexes were used in the screening, one at $\mathrm{pH} 4.5$ and the other at $\mathrm{pH} 8.5$ (later optimized to $\mathrm{pH} 9.0$ ). These solutions were diluted accordingly to compensate for the lower solubility of the 2-45 peptide complex. The protein drops that varied in size from 1.2 to $3.6 \mu \mathrm{l}$ were placed on the sitting-drop coverglass in the Q-plate setup (Stura, 2001) before layering the precipitant on top without mixing. Different volumes of protein and precipitant drops were used to control the rate of equilibration and the final protein concentration in the drop and to slow down equilibration, an important factor when crystallizing in system with high aggregation rates. Two rounds of crystal improvement by streak-seeding followed by macroseeding (Stura, 1999b) were added to improve crystal quality. The same procedure was used for the core 120 complex.

\subsection{Data collection}

Diffraction data were collected on beamlines ID14-EH2 and EH1 ID29 and BM30A (FIP) at the European Synchrotron Radiation Facility (ESRF) for Fab' 19D9D6 complex crystals transferred into a cryoprotectant solution containing $27 \%$ ethylene glycol; $13.8 \%(w / w)$ MPEG 5000 and $1.6 \mathrm{mM} \mathrm{ZnCl}, 1.6 \mathrm{mM} \mathrm{CdCl}, 55 \mathrm{~m} M$ sodium cacodylate $\mathrm{pH} 6.5$ and flash-frozen in the cryostream or in liquid ethane. All data were processed using the $H K L$ package (Otwinowski \& Minor, 1997).

\subsection{Structure determination and refinement}

Several crystals were grown and analyzed and data were collected. Each data set was solved by molecular replacement using $A M o R e$ (Navaza, 1994) with either the free Fab' 19D9D6 (PDB code 1nlb; Ménez et al., 2003) or the single-site PpL-Fab' 19D9D6 complex (PDB code 1mhh; Graille et al., 2002) taken as the model. The molecular replacement was followed by rigid-body refinement and cycles of conjugate-gradient and anisotropic temperature-factor refinement using CNS (Brünger et al., 1998) with the maximumlikelihood protocol. Progress was judged by the decrease of the free $R$ value. Electron-density maps (omit $\sigma_{\mathrm{A}}$-weighted $2 F_{\mathrm{o}}-F_{\mathrm{c}}$ and $\left.F_{\mathrm{o}}-F_{\mathrm{c}}\right)$ were calculated and displayed using the XtalView suite of programs (McRee, 1999) and TURBO (Roussel \& Cambillau, 1989). In the refinement, the complementarity-determining regions of the antibody were deleted and rebuilt to remove model bias in the region of the antigen-binding site. The peptide was modelled once the Fab' had been rebuilt.

\subsection{Structure analysis}

The final structures were analyzed with PROCHECK (Laskowski et al., 1993) and found to conform to standard stereochemistry. Statistics are reported in Table 1. 


\section{conference papers}

\section{Results and discussion}

\subsection{Crystallization}

The first crystallization attempts with $\mathrm{Fab}^{\prime}$ 19D9D6 and core peptides $2-45$ and $25-45$ yielded crystals that did not contain peptide. The strong tendency of these peptides to aggregate is believed to be the likely cause. This inconvenience was not known at the time. Thus, when further screening attempts were carried out they included the addition of various mutants of PpL. Crystals or microcrystals, were obtained either spontaneously or after seeding for most of the conditions and for all the mutants of PpL tested. Unfortunately, as in the absence of $\mathrm{PpL}$, some forms still contained no peptide. Others could not be grown to give diffraction-quality crystals and some crystals that diffracted were strongly anisotropic and could not be used for structure determination. By slowing down nucleation and crystal growth and using selected mutants of $\mathrm{PpL}$, three data sets could be collected with peptide $2-45$ present in the lattice and one data set for crystals grown in the presence of core 120.

\subsection{Crystals with peptide $2-45$}

At $\mathrm{pH} 4.5$, crystals grown from a solution containing peptide 2-45, Fab' 19D9D6 and PpL double mutant D55A + Y64W had no peptide in the lattice. However, by using another mutant L57H + D55A + Y64W and 9\% MPEG 5000 in $100 \mathrm{~m} M$ NaOAc instead of $10 \%$ MPEG 5000, crystals in space group $P 2_{1}$ with $a=43.6, b=230.5$, $c=123.6 \AA$ were obtained. These crystals contained four Fab and four PpL molecules but only three peptides in the asymmetric unit. Two peptide chains were bound to their specific Fab, while the third was almost completely disordered being threaded between the binding sites of the remaining two Fab in the asymmetric unit (Fig. 1a). The additional $\mathrm{L} 57 \mathrm{H}$ mutation weakens $\mathrm{PpL}$ site 1 binding (Housden et al., 2004) and thus it may help slow down the crystallization. A reduction in MPEG concentration is also implemented to achieve the same goal.

Another crystal form with similar unit-cell parameters but in the higher symmetry space group $P 2_{1} 2_{1} 2(a=129.5, b=222.5, c=43.8 \AA)$ was obtained in complex with the same PpL mutant but from a reservoir solution containing $8 \%$ MPEG 5000 in acetate buffer. These crystals diffract to $3.05 \AA$, contain two Fab's, two PpL molecules and two peptides in the asymmetric unit and no peptide is shared between different Fabs (Fig. 1b). The lower MPEG concentration may allow for a longer equilibration time before nucleation and this may have improved the homogeneity and the diffraction of the crystals.

A further improvement in resolution was achieved with $\mathrm{PpL}$ mutant $\mathrm{H} 74 \mathrm{C}$ and by shifting the crystallization $\mathrm{pH}$ (10.3\% MPEG $5000,500 \mathrm{~m} M \mathrm{NaCl}, 200 \mathrm{~m} M$ Tris-HCl pH 9.0). The higher $\mathrm{pH}$ was used to encourage disulfide-bond formation as in the crystallization of the disulfide-dimerized peptide in complex with its antipeptide antibody (Stura, Tête-Favier et al., 2002). However, the crystals obtained adopt the same lattice $\left(P 2{ }_{1} 2{ }_{1} 2\right.$ with $a=129.3, b=222.9$, $c=43.6 \AA)$ as those grown in acetate buffer at the lower $\mathrm{pH}$.

In this case instead of using a lower precipitant concentration to reduce the effect of the aggregated peptide on the crystallization, $\mathrm{NaCl}$ was added to diminish aggregation itself. In this way the MPEG concentration could be increased expecting that this would reduce the unit-cell volume and increase the diffraction limit of the crystals as discussed in Stura et al. (1999). The resultant crystals diffract to $2.59 \AA$

The unit-cell parameters of the monoclinic and orthorhombic space groups are very similar: the $\beta$-angle of the monoclinic cell is close to $90^{\circ}\left(91.7^{\circ}\right)$ and the packing in the two lattices is related. However, there is an important difference in the content of the asymmetric unit. To be identical to the orthorhombic form, the $P 2_{1}$ cell should contain four $\mathrm{Fab}^{\prime}$, four peptides $2-45$ and four $\mathrm{PpL}$ molecules. This is not the case. The binding sites of two of the four $\mathrm{Fab}^{\prime}$ come into close proximity of each other, peptide 2-45 is located in between their binding sites so that it can be considered to be 'shared' (Fig. 1a). In the crystal structure it is not possible to attribute the peptide between the two binding sites to either of the two approaching Fabs. It is likely to be a superposition, of no-peptide, a peptide bound on one of the Fab's with possibly some residues bound to the other. The arrangement is interesting as a curiosity regarding competition between antibodies for the same antigen, but because the resultant density is relatively non-specific is otherwise of little immunological interest. However, from a crystallization point of view, it highlights one of the consequences of crystallizing in the presence of a peptide or protein with a strong tendency to aggregate. Because of aggregation, there is insufficient peptide in solution for all the Fab's that is in the supersaturated state; thus some Fab's have to share. Although this appears to be an unlikely explanation, strangely, this is
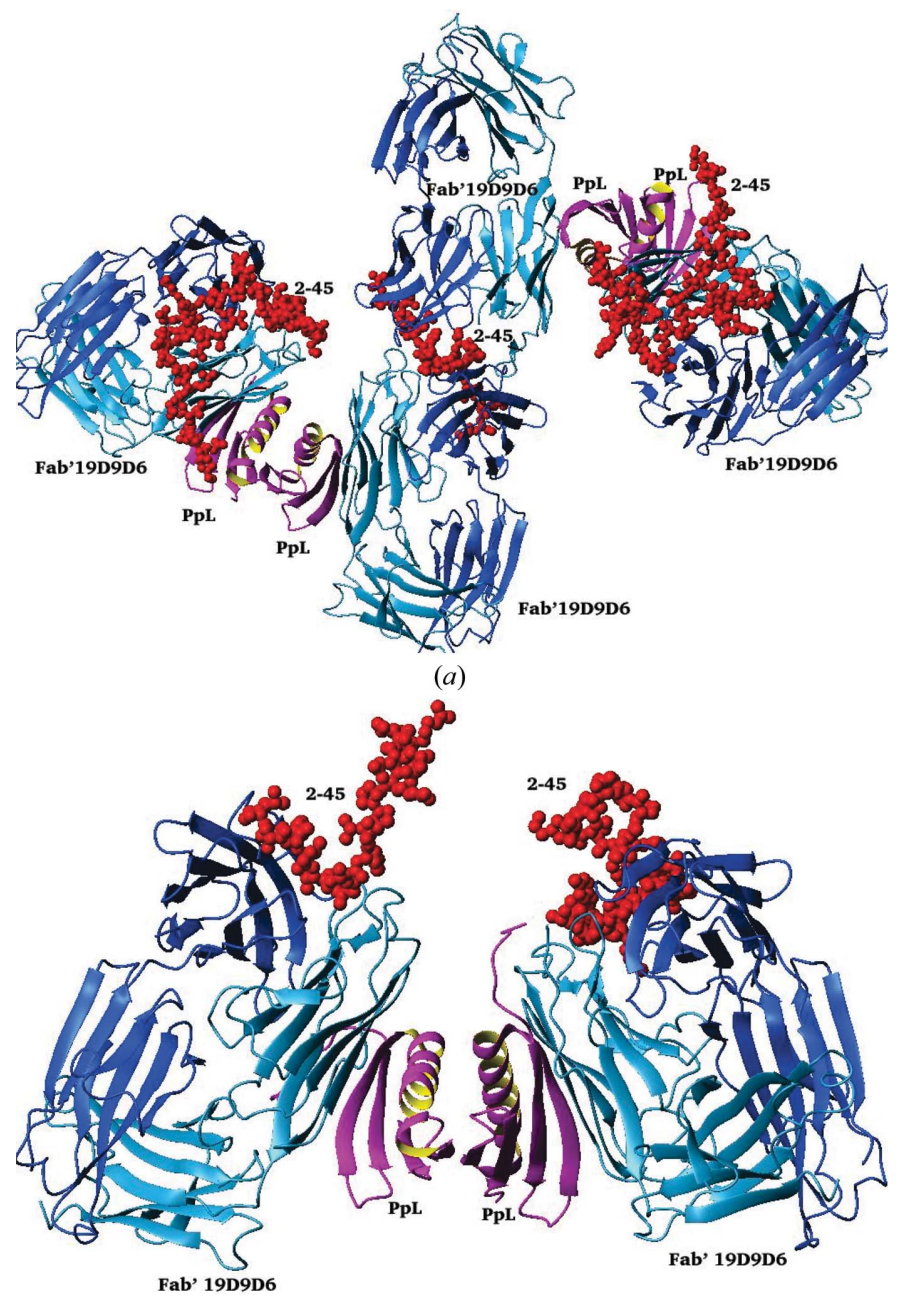

(b)

Figure 1

Comparison of the placement of the hepatitis C virus (HCV) core peptide 2-45 (red) in the lattice formed by the anti-HCV monoclonal antibody 19D9D6 (blue) and $\mathrm{PpL}$ (magenta) in the monoclinic $P 2_{1}(a)$ and in the orthorhombic space group $P 2_{1} 2_{1} 2(b)$. In the situation above two peptides are ordered but the peptide sandwiched between two antigen-binding sites is disordered. In the $P 2_{1} 2_{1} 2$ lattice all peptides become ordered. Peptide aggregation is the possible cause of the problem in the $P 2_{1}$ space group. Colour versions of the figures in this article are available in the online edition of the journal. 
what appears to be the case. Increasing the stoichiometric amount of peptide does not produce the desired result as there is already an excess of peptide and as more peptide is added it will join the peptide already in the aggregated state. Only by reducing the precipitant concentration from $9 \%$ MPEG to $8 \%$ can we slow down the crystallization and obtain a completely complexed $\mathrm{Fab}^{\prime}$. In order to accommodate the additional peptide the lattice must change. This leads to the change in space group.

\subsection{Crystals with recombinant HCV Core 120}

$\mathrm{PpL}$ mutant $\mathrm{H} 74 \mathrm{C}$ was also instrumental in obtaining crystals in the presence of the recombinant protein core120 in space group $P 2$ with $a=94.7, b=43.2, c=104.6 \AA, \beta=117.85^{\circ}$ with just one complex in the asymmetric unit. This occurrence is a relatively rare for this space group (Wukovitz \& Yeates, 1995). There is a disulfide bridge between the mutated cysteines that is made across the $P 2$ crystallographic symmetry operation and this may explain the occurrence of the rare $P 2$ space group. Unfortunately, like in the case of the monoclinic crystal form with peptide 2-45 the two Fabs are positioned with their binding sites facing each other in the lattice and the recombinant $\mathrm{HCV}$ core protein becomes symmetrically averaged and cannot be interpreted. The problem will be harder to solve than for the $2-45$ peptide as the tendency of the recombinant core 120 protein to aggregate is so much greater. The crystallization conditions already include $\mathrm{NaCl}$ to reduce aggregation and an even lower MPEG concentration.

\subsection{Overall structure of $\mathbf{2}-45$ peptide}

With the exception of the peptide 'shared' between two Fab's in the monoclinic space group (Fig. 1a), the conformation of the portion of the peptide in the antigen-combining site is indistinguishable from that found in the structure obtained for the shorter 13-40 peptide crystallized without PpL. Briefly, the peptide conformation is a wide bend with the QIVGG segment buried deepest in the antigencombining site between the $\mathrm{V}_{\mathrm{L}}$ and $\mathrm{V}_{\mathrm{H}}$ domains and interacting primarily with the hypervariable loops $\mathrm{L} 1, \mathrm{~L} 3$ and $\mathrm{H} 1, \mathrm{H} 2, \mathrm{H} 3$ but not with L2. The contributions of the heavy and light chains to binding are roughly equivalent. The peptide conformation is stabilized both by interactions with the antibody and by intra-peptide bonds. The interactions are mainly non-polar in character and the QIVGG stretch interacts via hydrogen bonds with the hypervariable regions of the $\mathrm{Fab}^{\prime}$.

\subsection{Structure of $2-45$ peptide in space group $P 2_{1}$}

The final electron density in space group $P 2_{1}$ for the trimolecular complex of the 2-45 peptide with PpL mutant D55A/L57H/Y64W and $\mathrm{Fab}^{\prime}$ 19D9D6 allows the positioning of three distinct peptide chains. Residues 17-40 for chain $P$ and chain $Q$, and 2-45 for chain $S$ can be positioned. Peptide $P$ is 'sandwiched' between two Fabs (Fig. 1a), its surface areas buried upon binding are $436 \AA^{2}$ with chain $A$ (Fab' light chain), $804 \AA^{2}$ with chain $B$ (Fab' heavy chain), $369 \AA^{2}$ with chain $E$ (Fab' light chain) and $794 \AA^{2}$ with chain $F$ (Fab' heavy chain), giving a total of $2402 \AA^{2}$. This surface represents the loss of accessibility of the surface to water molecules calculated using a $1.4 \AA$ probe in the program AREAIMOL.

Peptide $Q$ is bound to the antibody (chains $C$ and $D$ ) through eight hydrogen bonds, four involving the light chain and four the heavy chain. Residues Thr11, Asn14 and Gly33 interact with Asn27d, Ser56 and Gly57 of light chain $C$, and Gly26, Gln29 and Gly32 interact with Asp31, Asn52, Thr52a and Gln99 from heavy chain D. Finally, 16 intra-peptide interactions involving 20 residues stabilize the conformation.

Peptide $S$ is bound to the antibody (chains $G$ and $H$ ) by 11 hydrogen bonds, five involving Lys10, Lys12, Arg13, Asn14 and Gly33 interacting with Glu61, Ser67, Gly63 and Ala97 of the light chain, and six involving Gly28, Gln29 and Gly33 interacting with Asp31, Asn52, Thr53 and Gln103 of the heavy chain. Ten intra-peptide interactions among 16 amino acids of peptide $S$ determine the final peptide conformation.

\subsection{Structure of $2-45$ peptide in space group $P 2{ }_{1} 2_{1} 2$}

The final electron-density maps for the trimolecular complex between PpL mutant D55A/L57H/Y64W, 19D9D6 Fab' and the 2-45 peptide in space group $P 2{ }_{1} 2{ }_{1} 2$ at $3.05 \AA$ resolution allows the positioning of two peptides in the asymmetric unit (chains $P$ and $Q$ ). For chain $P$, residues $16-45$ can be placed in the electron density, but only residues $24-45$ from chain $Q$ can be fitted.

In addition to the specific interactions made with the antibody recognition site (Ménez et al., 2003), the peptides establish some nonspecific interactions with the immunoglobulin outside the binding site so that the surface area buried upon binding of peptide $P$ are $701 \AA^{2}$ with the light chain and $1155 \AA^{2}$ with the heavy chain for a total buried surface of $1856 \AA^{2}$. For peptide $Q$, only $542 \AA^{2}$ are buried with the light chain and $788 \AA^{2}$ with the heavy chain giving a total of $1328 \AA^{2}$. In the orthorhombic space group the Fab' binding sites are further apart so that one peptide can bind to each. Since the unit-cell volume remains constant with effectively more peptide bound, this results in a reduction in the size of the cavity available for the bound peptides. Thus, in this space group the peptides have less space for movement. Contrary to expectations, this does not lead to a better defined conformation for the peptide, although the crystals diffract to higher resolution.

Peptide $P$ is bound to the antibody (chains $A$ and $B$ ) through 11 hydrogen bonds, four involving the light chain $(A)$ and seven the heavy chain $(B)$. These interactions involved Ala16, Arg17; Gln29, Gly32, Gly33, Val34 and Ala44 for the peptide $P$, and Asn27d and Ala91 of the light chain, Tyr92, Thr52a, Glu56 and Gln99 from the heavy chain. Finally, 11 intra-peptide hydrogen bonds involving 14 residues stabilize the conformation.

Peptide $Q$ and antibody chains $C$ and $D$ are less well defined. Thus only two hydrogen bonds with light chain $\mathrm{C}$ and two with heavy chain $D$ involving residues Gly32 and Leu36 for $Q$ and Ala91C, $\operatorname{Arg} 27 f C$ and Gln99D for the antibody are formed. Only one intra-peptide hydrogen bond between Gly41 and Arg43 stabilizes the conformation.

\subsection{Epitope conformation in solution}

The conformation of peptide $2-45$ has been characterized in solution (PDB code 1cwx). It folds into two helices which pack against each other masking a small hydrophobic core. This structure is different from that adopted when bound to mAb 19D9D6. In the crystal structure, the peptide conformation is stabilized by interactions with the antibody, intra-peptide hydrogen bonds and several van der Waals interactions. In comparison to the solution structure, only a short stretch of five residues (32-26, GGVYL) is maintained.

Contrary to common sense, the larger cavity accommodates a better ordered peptide, suggesting that because in the smaller cavity it can establish a larger number of non-specific discrete interactions with surrounding residues, none of which are strongly predominant, the peptide becomes invisible in the electron density while in the 
larger cavity it can fold on itself and is less perturbed by non-specific contacts with the walls of the cavity.

\subsection{PpL dimerization}

In all the complexes described here $\mathrm{PpL}$ is dimeric. In the complex with core 120 and the mutated $\mathrm{H} 74 \mathrm{C}$, the dimer is formed by a disulfide between the mutated cysteines. In the complexes with the 2-45 peptide, the dimeric interface involves residues from the helical stretch $43-52$ and sheet residues $63-75$. At the centre of the interaction we find a symmetric hydrogen bond formed between the carbonyl oxygen of Ala66 with the main-chain nitrogen of Leu68, there is a salt bridge linking Arg52 and Asp44 and the rest of the interactions are mainly hydrophobic. The main residues involved in the hydrophobic core are Phe43, Tyr51, Arg52, Trp64, Thr65, Leu68, Gly71, Gly72. Of these residues W64 is mutated from the wild-type $\mathrm{PpL}$ and is involved in a crystal contact even in the absence of an antigen (Granata et al., 2005).

\subsection{Conclusions}

We had shown by competition ELISA experiments that in solution peptide 2-45 aggregates at high concentrations (Ménez et al., 2003). Aggregation can also be monitored by dynamic light-scattering (DLS) experiments, but this method was thought not to be appropriate in our case considering that the signal in DLS is proportional to the sixth power of the radius of gyration and the signal from an aggregated peptide could be smaller than that of the whole Fab' if the size of peptide aggregates did not exceed $50 \mathrm{kDa}$. Since the competition ELISA experiments clearly show that peptide aggregation is peptide-concentration dependent and in order to crystallize the complex between Fab' 19D9D6 and peptide 2-45 we needed high peptide concentrations, we expected to encounter aggregation and had to find a way to circumvent the problem. Given that aggregation is peptide-concentration dependent, as peptide becomes incorporated into the crystal of the complex, more should move out of its aggregated state into solution so that the complex crystals can continue to grow. Thus, by slowing down the crystallization, by simply reducing the precipitant concentration, we thought that we could grow complex crystals that would contain the $2-45$ peptide as long as

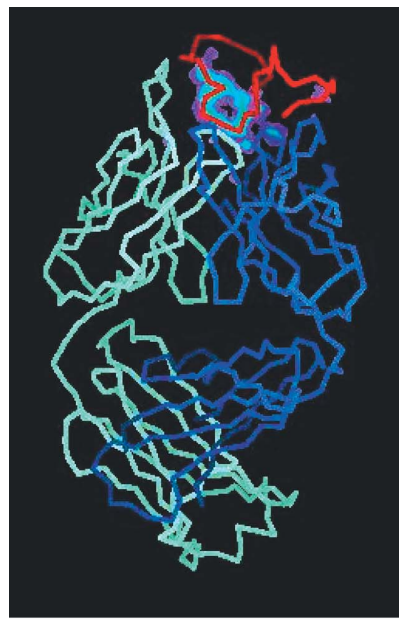

(a)

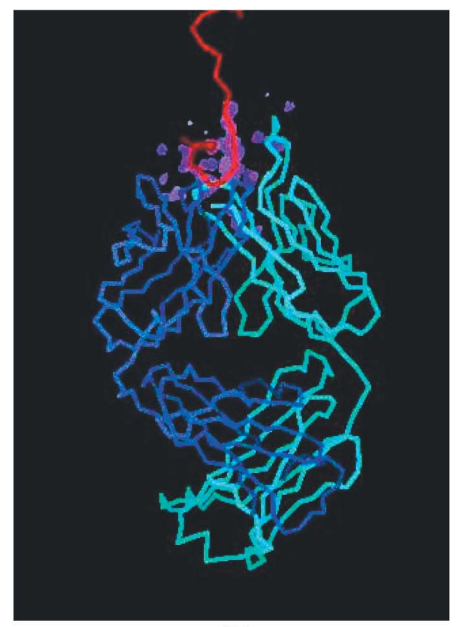

(b)
Figure 2

Omit map of peptide 2-45 in the complex with Fab' 19D9D6 (blue) and PpL (not shown). The two peptides (red) do not adopt the same conformation and are not as well ordered within and outside the recognition site. Peptide $P(a)$ is fully occupied and ordered while peptide $Q(b)$ is not, possibly because of poor peptide availability. peptide aggregation was reversible. Indeed, as the drops were set up we noticed that peptide or Fab-peptide complex fell out of solution, something that does not happen in the absence of the peptide. Its morphology was non-uniform and thus we considered this to be aggregated material rather than a classical precipitate. Later, crystals appeared and the structure showed that the objectives had been met.

However, the success must be attributed to the combination of slowing down equilibration and the use of specific mutants. Only two mutants gave usable data even if crystals were also obtained with other mutants. The single-site mutant $\mathrm{L} 57 \mathrm{H}$ that was shown by calorimetry and stopped-flow fluorescence to have reduced site 1 affinity (Housden et al., 2004) gives peptide-complexed crystals at low $\mathrm{pH}$, while PpL mutant $\mathrm{H} 74 \mathrm{C}$, that can bind at both site 1 and site 2, gives the same packing but at high $\mathrm{pH}$ with $\mathrm{NaCl}$ added. This may be due to the His to Cys mutation, but in order to separate out all the possibilities more trials would be needed.

How does the lattice affect the conformation of the peptide? Given that the peptide is semi-disordered with its ordered portion interacting with the $\mathrm{Fab}^{\prime}$ and the rest having minimal interactions with the lattice, the peptide can hardly affect crystal packing. The lattice does not influence the peptide conformation again because of lack of interactions. However, the size of the cavity created by the lattice influences the peptide structure by giving the peptide the space that it needs so it can adopt its lowest energy conformation.

When is slow slow enough? Aggregation does not prevent crystallization but it is a difficult problem to solve. In this case the lower MPEG concentration may have allowed for slower growth, may have improved the homogeneity and the diffraction of the crystals, but peptide incorporation in the crystals was not uniform. The electron density for peptides $P$ and $Q$ are quite different (Fig. 2). Slow growth helped, but it was not slow enough. Thus, when possible it is best to avoid aggregation by using a more soluble peptide. This may mean a shorter or a modified peptide that still retains full biological activity. This work may serve as a guideline to those that must grow crystals with aggregated material.

We thank the staff at the European Synchrotron Radiation Facility (ESRF) in Grenoble on beamlines ID14, ID29 and BM30A (FIP) for their assistance. We thank Stéphane Bréssanelli and Marie-Hélene Le Du for help in collecting data and Marc Artigue (BioTom SA) for financial support.

\section{References}

Brünger, A. T., Adams, P. D., Clore, G. M., DeLano, W. L., Gros, P., GrosseKunstleve, R. W., Jiang, J.-S., Kuszewski, J., Nilges, M., Pannu, N. S., Read, R. J., Rice, L. M., Simonson, T. \& Warren, G. L. (1998). Acta Cryst. D54, 905-921.

Graille, M., Harrison, S., Crump, M. P., Findlow, S. C., Housden, N. G., Muller, B. H., Battail-Poirot, N., Sibaï, G., Sutton, B. J., Taussig, M. J., JolivetReynaud, C., Gore, M. G. \& Stura, E.A. (2002). J. Biol. Chem. 277, 4750047506.

Granata, V., Housden, N. G., Harrison, S., Jolivet-Reynaud, C., Gore, M. G. \& Stura, E. A. (2005). Acta Cryst. D61, 750-754.

Housden, N. G., Harrison, S., Housden, H. R., Thomas, K. A., Beckingham, J. A., Roberts, S. E., Bottomley, S. P., Graille, M., Stura, E. \& Gore, M. G. (2004). J. Biol. Chem. 279, 9370-9378.

Jolivet-Reynaud, C., Dalbon, P., Viola, F., Yvon, S., Paranhos-Baccala, G., Piga, N., Bridon, L., Trabaud, M. A., Battail, N., Sibai G. \& Jolivet, M. (1998). J. Med. Virol. 56, 300-309.

Ladavière, L., Deléage, G., Montserret, R., Dalbon, P., Jolivet, M. \& Penin, F. (1997). Proceedings of the Fourth International Meeting on Hepatitis C Virus and Related Viruses, Kyoto, Japan.

Laskowski, R. A., MacArthur, M. W., Moss, D. S. \& Thornton, J. M. (1993). J. Appl. Cryst. 26, 283-291.

McRee, D. E. (1999). J. Struct. Biol. 125, 156-165. 


\section{conference papers}

Ménez, R., Bossus, M., Muller, B. H., Sibai, G., Dalbon, P., Ducancel, F., Jolivet-Reynaud, C. \& Stura, E. A. (2003). J. Immunol. 170, 1917-1924.

Navaza, J. (1994). Acta Cryst. A50, 157-163.

Otwinowski, Z. \& Minor, W. (1997). Methods Enzymol. 276, 307-326.

Park, H. J., Byun, S. M., Ha, Y. J., Ahn, J. S. \& Moo, H. M. (1995). J. Immunoassay, 16, 167-181.

Roussel, A. \& Cambillau, C. (1989). Silicon Graphics Geometry Partners Directory, pp. 77-78. Silicon Graphics, Mountain View, CA, USA.

Stura, E. A. (1999a). Crystallization of Proteins: Techniques, Strategies and Tips. A Laboratory Manual, edited by T. Bergfors, p. 113. International University Line.
Stura, E. A. (1999b). Crystallization of Nucleic Acids and Proteins: A Practical Approach, 2nd ed., edited by A. Ducruix \& R. Giegé, p. 177. Oxford University Press.

Stura, E. A. (2001). J. Cryst. Growth, 232, 545-552.

Stura, E. A., Charbonnier, J.-B. \& Taussig, M. J. (1999). J. Cryst. Growth, 196, $250-260$.

Stura, E. A., Martin, L., Lortat-Jacob, H., Vivès, R. \& Vita, C. (2002). Acta Cryst. D58, 1670-1673.

Stura, E. A., Tête-Favier, F., Muller, S. \& Aubry, A. (2002). Acta Cryst. D58, 1740-1743.

Wukovitz, S. W. \& Yeates, T. O. (1995). Nature Struct. Biol. 2, 1062-1067. 